\title{
Multichannel thermography systems for real-time and transient thermal process application
}

\author{
by B. Więcek and S. Zwolenik
}

Technical University of Łodż, Institute of Electronics, Computer Thermography Group 18/22

Stefanowskiego St, 90-924 Łódż, Poland, e-mail: wiecek@ck-sg.p.lodz.pl, zwolenik@ck-sg.p.lodz.pl

\begin{abstract}
This paper presents the architecture, software and applications of high-speed, multichannell thermal and visual computer systems based on PCl (Peripheral Component Interconnect) and PCMCIA standards. The presented solutions are general and can be applied both for various cameras and computer systems. The new image processing algorithms were implemented to ensure more precise temperature measurements, e.g.: 2D perspective transformation, directional emissivity correction, bicubic interpolation, etc.
\end{abstract}

\section{Introduction}

An interface links up to 2 CCD color cameras and 1 thermal camera with the powerful computer. The interface is the Peripheral Component Interconnect (PCl) plug-in card offering the high performance 32-bit data transfer and optional burst mode that provides accelerated throughput of data across the bus of $1.32 \mathrm{MB} / \mathrm{s}$ [3]. There are many systems available for thermal and optical image processing, but most of them work separately, often off-line, without powerful tools to. process thermal and visual images in parallel [1-2]. The system presented in this work is one of the first providing enough data transfer throughput to capture and process images in real-time. A software which co-operates with the interface is helpful for more advanced application, wherever the 2-D and 3-D operations are required.

\section{System overview - hardware}

A system contains of analog and digital part as shown in Fig. 1. In the analog part the new video processor is applied with two 8-bit AVD converters and synchronisation circuit. The CCD flash ADC is on-chip equipped with 2-input analog multiplexers. It makes possible to capture visual images one-by-one, with the delay of one frame (20ms), but in parallel with thermal image. In the digital part $1 \mathrm{MB}$ buffer VRAM memory is used to store images and transfer them to the computer. A dual-port memory is suitable for fast data acquisition, and it allows easy to synchronise the data capturing with its transfer to the operational memory. Entire control system is integrated in high density FPGA (Field Programmable Gate Array), providing high flexibility of the design and possibility of system reconfiguration for different cameras. Actually, our system is prepared for thermal cameras with both analog and digital outputs.

Sampling clock restoration is one of the most important elements of the system. There are two independent clock recovery blocks for video and thermal channels, however because of high speed of data conversion for CCD camera, this sampling clock circuitry is more difficult to implement and has to be fit in high-speed external PAL. Sampling rate of video signal (CCIRT-PAL) at $14.75 \mathrm{MHz}$ ensures the square pixel and the proper image size on the screen. Video capturing circuit works with clock restoration using the Digital Phase Lock Loop circuitry (DPLL). This causes the high stability of the restored sampling clock and in consequence the high quality of the captured image. DPLL-based restoration circuit is recommended in high-resolution video systems, where the slight changes in the phase clock can disturb the captured image smoothness.

The memory buffer implemented using fast dual-port memories (VRAM) is designed as a FIFO (First-In-First-Out) memory. Input data coming from AVD converters is loaded into the 
memory through Serial Register (SAM). The corresponding line counter for locating the memory row where the pixels are placed in is implemented in FPGA. This control block contains also similar counters for pixels and lines to read data from the memory and transfer them to the computer.

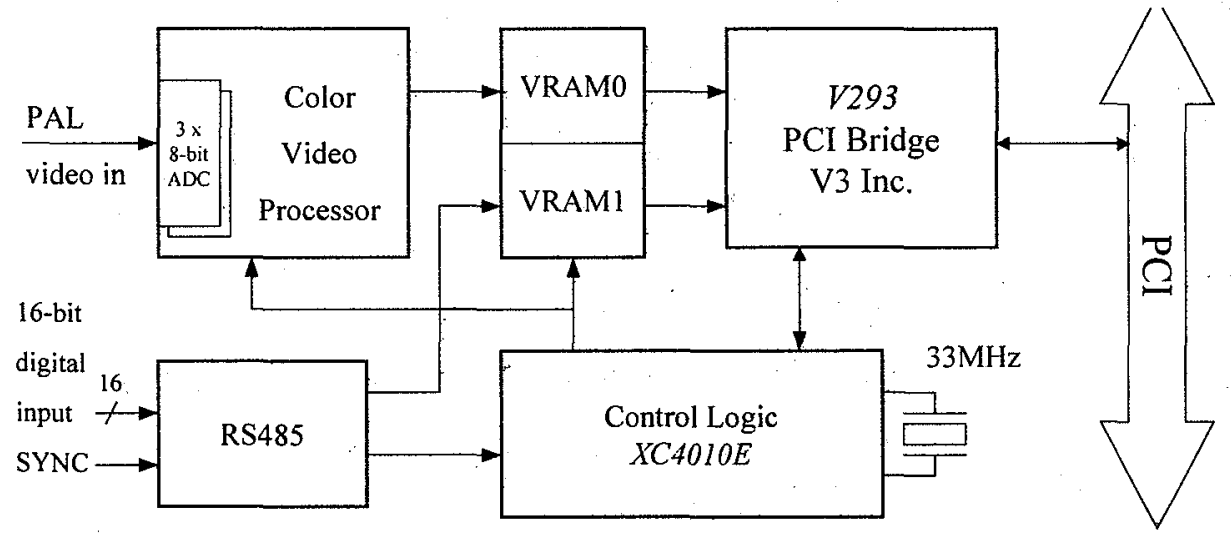

Fig.1. Hardware diagram of PCl interface

$\mathrm{PCl}$ is becoming a fundamental building block of today's high-performance $\mathrm{PC}$ and workstation peripherals, and developing products that meet the stringent $\mathrm{PCl}$ Local Bus Specification is the next challenge for system designers. The developed $\mathrm{PCl}$ interface provides the unique features helpful for real-time and transient thermal process investigations.

- Automatic configurations: PCl hosts automatically identify and configure devices, an important feature in Plug and Play applications. Automatic configuration eliminates the need for the configuration switches commonly found on Industry Standard Architecture (ISA) cards.

- Low power consumption: The $\mathrm{PCl}$ interface supports both 5.0V and 3.3V operation for easier migration from $5.0 \mathrm{~V}$ to $3.3 \mathrm{~V}$ environments. The $\mathrm{PCl}$ Interface also supports mixedvoltage environments.

- High-performance data transfer. PCl offers high-performance 32- or 64-bit data transfer and an optional burst mode that provides accelerated throughput of data across the bus. Device connected to the $\mathrm{PCl}$ interface achieves performance comparable to that of devices directly connected to the processor local bus.

\section{Advanced thermal and visual image processing}

The software for thermal and visual image processing to manipulate with thermal and video images and their sequences was written on Delphi and $\mathrm{C}++$ language and it is native 32-bit code. The software is Win'95 and WinNT compatible.

The software is dedicated for thermal and visual image processing in parallel. There are various functions available to measure temperature easy and precisely. Some of them are listed below in Table 1. All images, both thermal and visual ones can appear on the screen in so-called life-mode. The throughput of the entire system allows to display them in real-time, to adjust distance, focus, brightness, contrast, etc. There are typical operations are implemented on thermal and video images. Histogram equalisation, low and high-pass filtering, contours extraction, zooming are the most useful: 
Table 1: Chosen functions of thermal and video system

\begin{tabular}{|l|l|}
\hline - Calibrated temperature measurement & - Low-pass and high-pass filtering \\
- Histograms & - Contours extraction \\
- Thermal cross-sections & - Plane transformations \\
- Setting reference points & - Contrast enhancement \\
- Histogram equalisation & - Powerful tools - points, lines, polygons \\
\hline
\end{tabular}

Additionally, the presented software gives the ability of directional emissivity correction. This algorithm uses full information about 3D shape of the observed object computed on the base of pictures taken from 2 or more CCD cameras [1-2]. It allows computing the distance between camera and the object in order to measure temperature more precisely.

Some photogrammetry algorithms are implemented as well. A transformation of a surface in a central projection is one of the most useful. It allows collecting fragmented objects by taking into account the angle of viewing. Geometrically transformed images are easy to stick together and measure the real parameters of an object, e.g. an area, distance, etc.

Various software tools used well-known method for image gluing based on transformation, which uses the picture scaling, rotation and shifting - eq. (1). Such transform needs only 3 reference points defined on the plane.

$$
\left[\begin{array}{l}
X \\
Y
\end{array}\right]=\left[\begin{array}{ll}
a_{11} & a_{12} \\
a_{21} & a_{22}
\end{array}\right] \cdot\left[\begin{array}{l}
x^{\prime} \\
y^{\prime}
\end{array}\right]+\left[\begin{array}{l}
d_{1} \\
d_{2}
\end{array}\right]
$$

where: $a_{i i}$ - scaling and rotation coefficients, $d_{i}$ - shifting coefficients, $X, Y$ - co-ordinates after transformation, $x^{\prime}, y^{\prime}$ - co-ordinates of an original pixel.

This method is only valid if the original and transformed planes are in parallel, and therefore it uses only 3 reference points.

The more useful method can transform the surfaces with different orientation in the 3D space as shown in Fig. 2. This method needs 4 reference points, as it has 8 transform parameters as shown in eq. (2).

$$
\left\{\begin{array}{l}
X_{i}=\frac{u_{1} \cdot x_{i}^{\prime}+u_{2} \cdot y_{i}^{\prime}+u_{3}}{u_{7} \cdot x_{i}^{\prime}+u_{8} \cdot y_{i}^{\prime}+1} \\
Y_{i}=\frac{u_{4} \cdot x_{i}^{\prime}+u_{5} \cdot y_{i}^{\prime}+u_{6}}{u_{7} \cdot x_{i}^{\prime}+u_{8} \cdot y_{i}^{\prime}+1}
\end{array}\right.
$$

where: $u_{i}$ - transform coefficients; $X, Y$-co-ordinates after transformation (real co-ordinates); $X^{\prime}, y^{\prime}$ - co-ordinates of an original pixel.

Even better results can be obtained using more reference points and applying the least-square approximation method.

The results of 4-point projection are presented in Fig.3. The original upper image is a projection of a front building side on the plane not perpendicular to the camera optical axis. The windows shown above are not of the rectangular shape. After transformation of the front building plane on the plane normal to the camera axis the windows become rectangular, and the entire scene keeps the real aspect ratio, significantly reducing the perspective distortions. After such correction it is quite easy to stick together a set of images and present the whole scene in the orthogonal projection.

Another function of the software makes possible to overlay visual and thermal images. The assumption of such superimposing is that the thermal and visual images are projected on the same or parallel planes. We can easily wrap, shift and mix two images. 
http://dx.doi.org/10.21611/qirt.1998.051

Different approach for image superposing uses the visual contour to be mixed thermal image as shown in Fig. 3. It simplifies or in certain cases allows locating precisely the hot spots. Two images (thermal and optical) allow to use common cursor, indicating a certain point on CCD one, and read temperature on thermal one.

\section{Conclusion}

The system for thermal and visual image processing is presented in this work. It can work in different modes, capturing images in real-time, frame-byframe, or storing a sequence with defined interval between the following images. The software for the interface supports Windows95 and WinNT

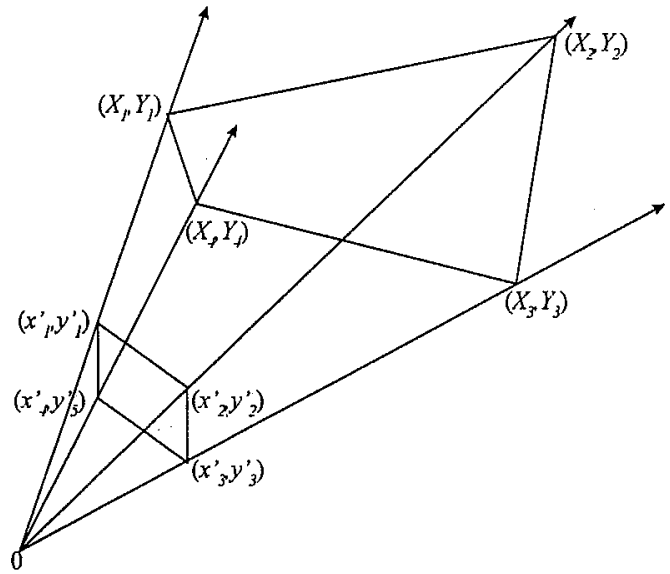

Fig.2. Plane-to-plane transform in central projection compatibility. The presented system is actually used and recommended for highly advanced thermal and visual images processing, e.g. 3-D photogrametry reconstruction, noise cancelling, image superimposing, etc., and improves the thermal camera measuring parameters.

\section{REFERENCES}

[1] WIECEEK (B.), GRECKI (M.). - Advanced Image Processing in Thermography. Proc. Conf. QUANTITATIVE INFRARED THERMOGRAPHY, Eurotherm Seminar 27. Sorento, 23-26 Aug. 1994, Italy.

[2] SITEK (Z.) - Fotogrametria ogólna i inżynieryjna. PPWK im. E. Romera, Warsaw, 1991, Poland (in Polish)

[3] WIECCEK (B.), ZWOLENIK (S.): - FPGA Approach to the PCI Bridge Design, Mixed Design of Integrated Circuits and Systems MIXDES'96, Łódź, Poland, 30 May - 1 June 1996 
http://dx.doi.org/10.21611/qirt.1998.051
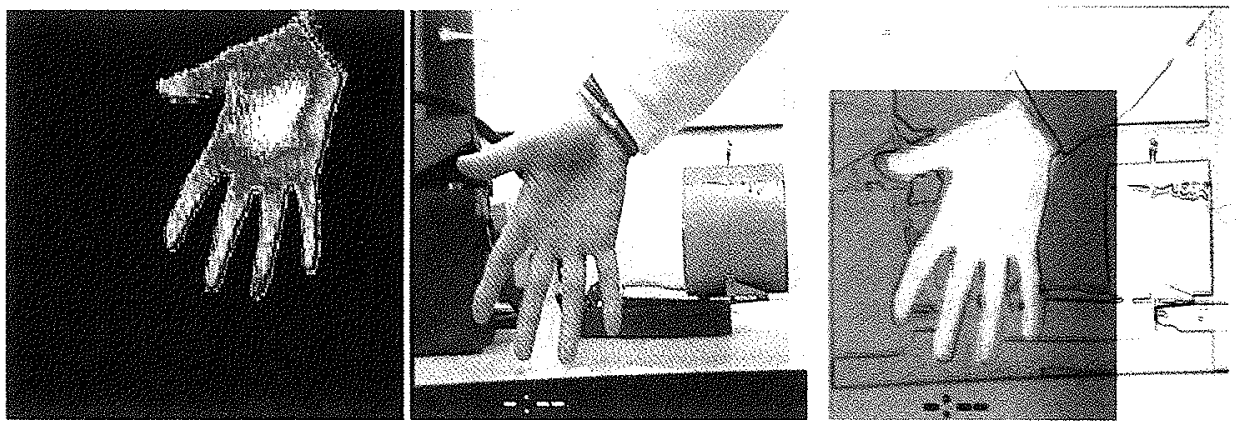

Fig. 3. Overlappying thermal and visual images
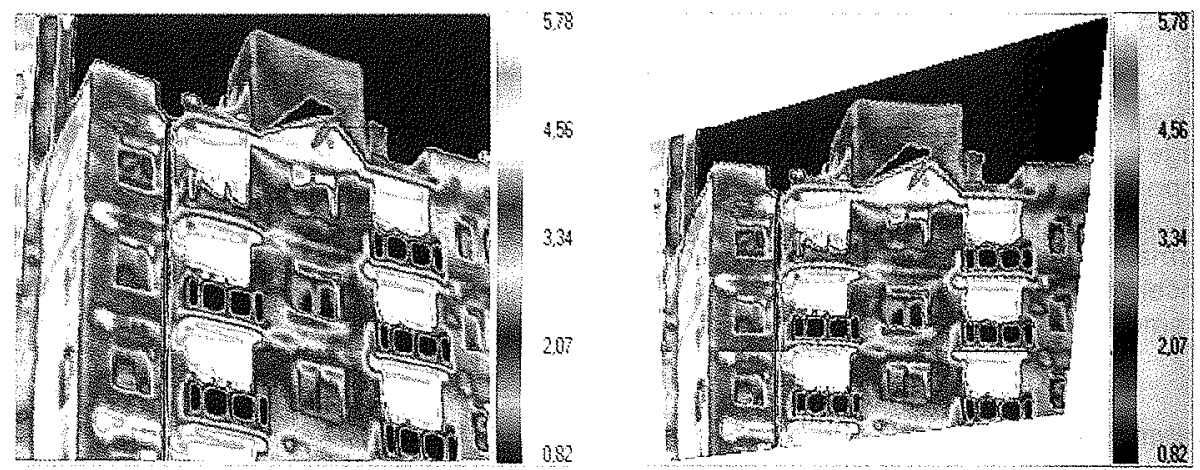

Fig. 4. 2D plane-to-plane transformation
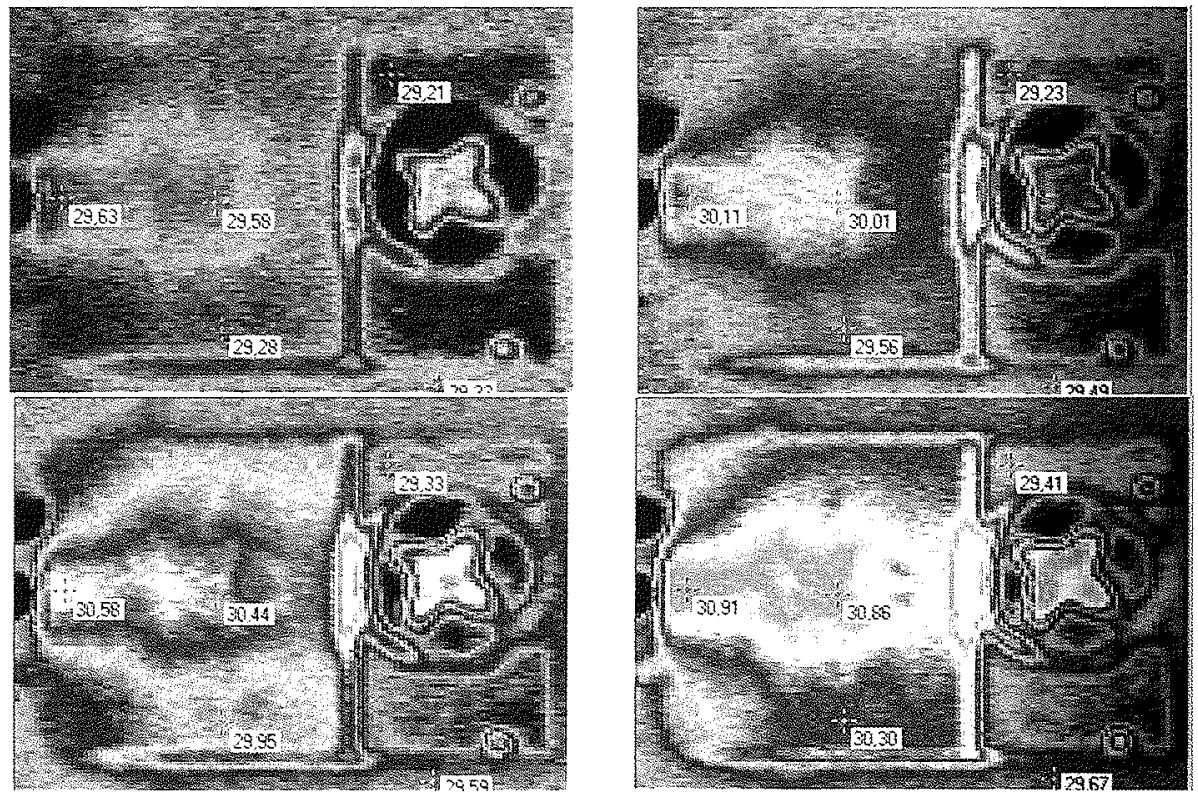

Fig. 5 Real time capturing (chosen frames from sequence of images) 